\title{
In vivo MRI assessment of the human Locus Coeruleus along its rostrocaudal extent in young and older adults
}

Matthew J Betts ${ }^{1,2}$, Arturo Cardenas-Blanco ${ }^{1,2}$, Martin Kanowski ${ }^{3}$, Frank Jessen ${ }^{4,5}$, Emrah Düzel ${ }^{1,2,6}$

${ }^{1}$ German Center for Neurodegenerative Diseases (DZNE), Magdeburg, Germany 2Institute of Cognitive Neurology and Dementia Research, Otto-von-GuerickeUniversity Magdeburg, Magdeburg, Germany

${ }^{3}$ Department of Neurology, University Hospital of Magdeburg, Otto-vonGuericke-University Magdeburg, Magdeburg, Germany

${ }^{4}$ German Center for Neurodegenerative Diseases (DZNE), Bonn, Germany

${ }^{5}$ Department of Psychiatry, University of Cologne, Cologne, Germany

${ }^{6}$ Institute of Cognitive Neuroscience, University College London, 17 Queen

Square, London, UK

Corresponding author: Dr Matthew J Betts

Corresponding author's address: Deutsches Zentrum für Neurodegenerative

Erkrankungen e.V. (DZNE) c/o Universitätsklinikum Magdeburg, Leipziger

Strasse 44, Haus 64, 39120 Magdeburg, Deutschland

Corresponding author's phone and fax: Tel. +49 391 67-25058; Fax +49 39167 -

25060

Corresponding author's e-mail address: matthew.betts@dzne.de

Conflict of interest: The authors declare no competing financial interests

Keywords: in vivo, brainstem, locus coeruleus, neuromelanin-sensitive MRI, aging 


\begin{abstract}
The locus coeruleus (LC), a major origin of noradrenergic projections in the central nervous system (CNS), may serve a critical role in the pathogenesis of neurodegenerative disorders such as Alzheimer's disease (AD) and Parkinson's disease (PD). As such, there is considerable interest to develop magnetic resonance imaging (MRI) techniques to assess the integrity of the LC in vivo. The high neuromelanin content of the LC serves as an endogenous contrast for MRI but existing protocols suffer from low spatial resolution along the rostrocaudal axis of the LC rendering it difficult to differentiate its integrity in caudal and rostral portions. This study presents a novel approach to investigate the human LC in vivo using $\mathrm{T}_{1}$-weighted Fast Low Angle Shot (FLASH) MRI at 3 Tesla (T). Using high-resolution isotropic imaging to minimise the effect of low spatial resolution in the slice direction, this study aimed to characterise the rostrocaudal distribution of LC signal intensity attributed to neuromelanin from 25 young (22-30) and 57 older (61-80) adults. We found a significant age-related increase in maximum but not median signal intensity, indicating age-related differences were not homogenous. Instead, they were confined to the rostral third of the LC with relative sparing of the caudal portion. The findings presented demonstrate in vivo $\mathrm{T}_{1}$-weighted FLASH imaging may be used to characterise signal intensity changes across the entire rostrocaudal length of the LC (a corresponding standardised LC map is available for download), which may help to identify how the human LC is differentially affected in aging and neurodegenerative disease.
\end{abstract}




\section{INTRODUCTION}

The locus coeruleus (LC) - one of the smallest but most extensively projecting nuclei in the upper dorsolateral pontine tegmentum of the brainstem, is responsible for more than half of the brain's cellular capacity of synthesised noradrenaline (NA) (Aston-Jones and Cohen, 2005; Espay et al., 2014). LC noradrenergic (LC-NA) innervation has been assigned putative roles in numerous central nervous system (CNS) functions such as working memory, learning and attention (Aston-Jones \& Cohen, 2005; Robbins, 2000; Benaroch, 2009), memory consolidation and retrieval (Sara, 2009; Sterpenich et al., 2006), vigilance, arousal/wakefulness, rapid eye movement (REM) sleep behaviour and pain modulation (Aston-Jones and Bloom, 1981; Benarroch, 2009; Espay et al., 2014).

It has been proposed that the LC plays a critical role in the progression of Alzheimer's disease (AD) whereby tau aggregates in the LC prior to the transentorhinal/entorhinal cortex and subsequent neocortex (Braak et al., 2011). Additionally in Parkinson's disease (PD), intraneuronal $\alpha$-synuclein burden in the LC precedes and may be of even greater magnitude than that in the substantia nigra pars compacta (SNpc) (Braak et al., 2003; Dickson et al., 2008). Furthermore, pathological changes in the LC are not exclusive to AD and PD, and have also been reported in Huntington's disease, progressive supranuclear palsy, Lewy body disease, Down's syndrome, multiple sclerosis and amyotrophic lateral sclerosis (for a review see Samuels and Szabadi, 2008a). Crucially, the topographical distribution of cell loss in the LC may vary across disorders whereby the most rostral portion may be more prone to greater cell loss than the caudal third as in AD and DS (Theofilas et al., 2016; German et al., 1992), and in contrast to the extensive neuronal loss across the entire length of the LC observed in PD (Vermeiren and De Deyn, 2017).

As reviewed by Mather and Harley (2016), earlier studies examining LC neuropathology in aging have suggested an age-related decline in LC neuron number by approximately 20-40\% (German et al., 1988; Lohr \& Jeste 1988; Marcyniuk et al., 1989; Tomlinson et al., 1981; Vijayashankar et al., 1979). However as noted by Mather and Harley (2016), a number of these studies made lifespan comparisons using very small sample sizes and did not account for additional pathology in the brain (German et al., 1988; Lohr and Jeste, 1988; Chan-Palay \& Asan, 1989) and more recent findings from cases free of additional brain pathology and/or using unbiased stereological procedures have found no age-related differences in the LC (Kubis et al., 2000; Ohm et al., 1997; Mouton et al., 1994; Theofilas et al., 2016). Furthermore age-related loss of LC cells may be regional rather than diffuse whereby greater cell loss has been reported in the rostral portion, with little to no cell loss in the caudal pole - a similar pattern of cell loss to that found in AD (Manaye et al., 1995; Theofilas et al., 2016). Additionally, there is clear evidence that tau pathology in the LC region increases with age (Braak et al., 2011; Braak and Del Tredici, 2015) and that age-related decreases in LC-mediated noradrenergic neurotransmission is related to lower cognitive function in older adults (Mather and Harley, 2016; Sara, 2009). Recent 
evidence would indicate maintaining the neural density of the LC-NA nuclei may prevent cognitive decline in aging (Wilson et al., 2013).

The LC contains large amounts of neuromelanin - a pigmented polymer that results from the oxidation of catecholamines including NA in the LC and dopamine in the SNpc (Wakamatsu et al., 2015; Zucca et al., 2006). Furthermore as people age, neuromelanin increases in the LC (Zucca et al., 2006). Neuromelanin may exert a neuroprotective role by chelating environmental toxins including heavy metals such as iron, cadmium and mercury to reduce toxicity but may also become neurotoxic when neuromelanin-containing neurons die and metals and toxic components previously accumulated are released into the extracellular space leading to chronic inflammation (Zucca et al., 2015). Crucially, neuromelanin itself serves as an endogenous magnetic resonance (MR) contrast agent and its relaxivity is strongly enhanced by binding metal ions, such as iron and copper (Enochs et al., 1989; Enochs et al., 1997; Trujillo et al., 2016), thereby providing a unique opportunity to visualise the LC region using neuromelanin-sensitive magnetic resonance imaging (MRI). Thus MR sequences optimized to detect neuromelanin provide an opportunity to probe the structure and function of the LC in vivo.

To date, the majority of studies using in vivo MRI to visualise the LC have used $\mathrm{T}_{1}$-weighted Turbo Spin Echo (TSE)/ Fast Spin Echo (FSE) imaging (GarciaLorenzo et al., 2013; Keren et al., 2009; Sasaki et al., 2006; Shibata et al., 2006; Takahashi et al., 2015). These studies used TSE/FSE sequences with a very high in-plane resolution and, therefore, required large slice thicknesses to maintain sufficient signal-to-noise ratio. In this study we introduce an alternative approach using three-dimensional high-resolution $\mathrm{T}_{1}$-weighted Fast Low Angle Shot (FLASH) MRI at 3 Tesla (T) to characterise the location and distribution of LC signal intensity distributions attributed to neuromelanin in young and older adults. By imaging the LC using isotropic resolution we aim to reduce the effect of low spatial resolution in the slice direction to assess the topographical distribution of signal intensity changes across the entire rostrocaudal extent of the LC. It is hoped the advancement of neuromelanin-sensitive MRI to investigate the role of the LC in vivo may help to elucidate why the LC is especially vulnerable to damage in neurodegenerative disease.

\section{METHODS}

\subsection{Study subjects}

25 young (22-30 years old; M:F 13:12) and 57 healthy elderly adults (61-80 years old; M:F 19:38, years of education: $16 \pm 2$ ) participated in the study. 35 elderly adults were recruited via the DZNE Longitudinal Cognitive Impairment and Dementia (DELCODE) study. With the exception of 2 elderly adults, all subjects recruited were right-handed. Written informed consent was obtained from all participants in accordance with the Declaration of Helsinki and the study received ethical approval from the Faculty of Medicine at the University of Magdeburg. All older adults were screened to ensure intact global cognitive 
performance using a brief neuropsychological battery (MMSE $29 \pm 1$ ), exclude neurological or major psychiatric illness and cases of white matter (WM) ischaemia. Prior to recruitment into this study, $\mathrm{T}_{2}$-weighted images acquired with 3T MRI were visually inspected to exclude severe brain abnormalities as previously described (Acosta-Cabronero et al., 2016). No subjects demonstrated evidence of micro-haemorrhages. Individuals known to have had a myocardial infarction, significant cardiovascular history or diabetes mellitus were not eligible for participation.

\subsection{Imaging protocol}

MRI measurements were performed on a Siemens Verio 3 Tesla system with a Siemens 32-channel phased-array head coil for reception. In order to visualise the LC, high-resolution $\mathrm{T}_{1}$-weighted Fast-Low-Angle-Shot (FLASH) images were acquired using the following parameters: $0.75 \times 0.75 \times 0.75 \mathrm{~mm}^{3}$ voxel size, $320 \times 320 \times 192$ matrix, $5.56 \mathrm{~ms}$ echo time, $20 \mathrm{~ms}$ repetition time, $23^{\circ}$ flip angle, $130 \mathrm{~Hz} /$ pixel bandwidth, 7/8 partial Fourier, and 13:50 min scan duration. The axial 3D block was oriented parallel to the floor of the $4^{\text {th }}$ ventricle.

$\mathrm{T}_{1}$-weighted MPRAGE anatomical images were also acquired in the same scanning session using the following acquisition parameters: $1 \times 1 \times 1 \mathrm{~mm}^{3}$ voxel size, $256 \times 256 \times 192$ matrix, $4.37 \mathrm{~ms}$ echo time, $2500 \mathrm{~ms}$ repetition time, $1100 \mathrm{~ms}$ inversion time, $7^{\circ}$ flip angle, $140 \mathrm{~Hz} /$ pixel bandwidth, $7 / 8$ partial Fourier, GRAPPA factor of 2 and 5:08 min scan duration.

A thin pillow was placed on the base of the coil surrounding the sides and the back of the head to guarantee an optimal balance between tight fixation and patient comfort as well as to minimise motion.

\subsection{Spatial standardisation and co-registration}

Prior to spatial standardisation, single-subject FLASH images were upsampled to a matrix size of $639 \mathrm{X} 639 \mathrm{X} 383$; equivalent to $(0.375 \mathrm{~mm})^{3}$ isotropic resolution, by convoluting with a sinc function in Matlab (2014b; Mathworks Inc., Natick, MA, USA). This matrix size was selected to improve visualisation in the LC region whilst maintaining sufficient boundary definition required for manual segmentation. Interpolating images to a larger matrix size resulted in poorer delineation of the LC from the surrounding region. RF-bias corrected with N4ITK sinc-interpolated FLASH images were standardised (to a study-wise space) by way of a parallel routine for template calculation provided in ANTs v2.1. Simultaneous co-registrations were driven by a cross-correlation minimisation performed over three resolutions with a maximum of 90 iterations at the coarsest level, 30 at the next coarsest and 90 at full resolution; template update step size was set to $0.1 \mathrm{~mm}$. One rigid-then-affine iteration was followed by eight full runs of the above non-linear multi-resolution routine to ensure stable convergence. The spatial standardisation, needed to generate the study-wise template was achieved through the warp composition of the above transformations and third-order b-spline interpolation. The LC and reference 
masks delineated in template space were warped to each subject in the studywise template using the 'WarpImageMultiTransform' command and nearest neighbour interpolation in ANTs v2.1 (Avants et al., 2008). Fig. 1 summarises the steps in the proposed processing pipeline.

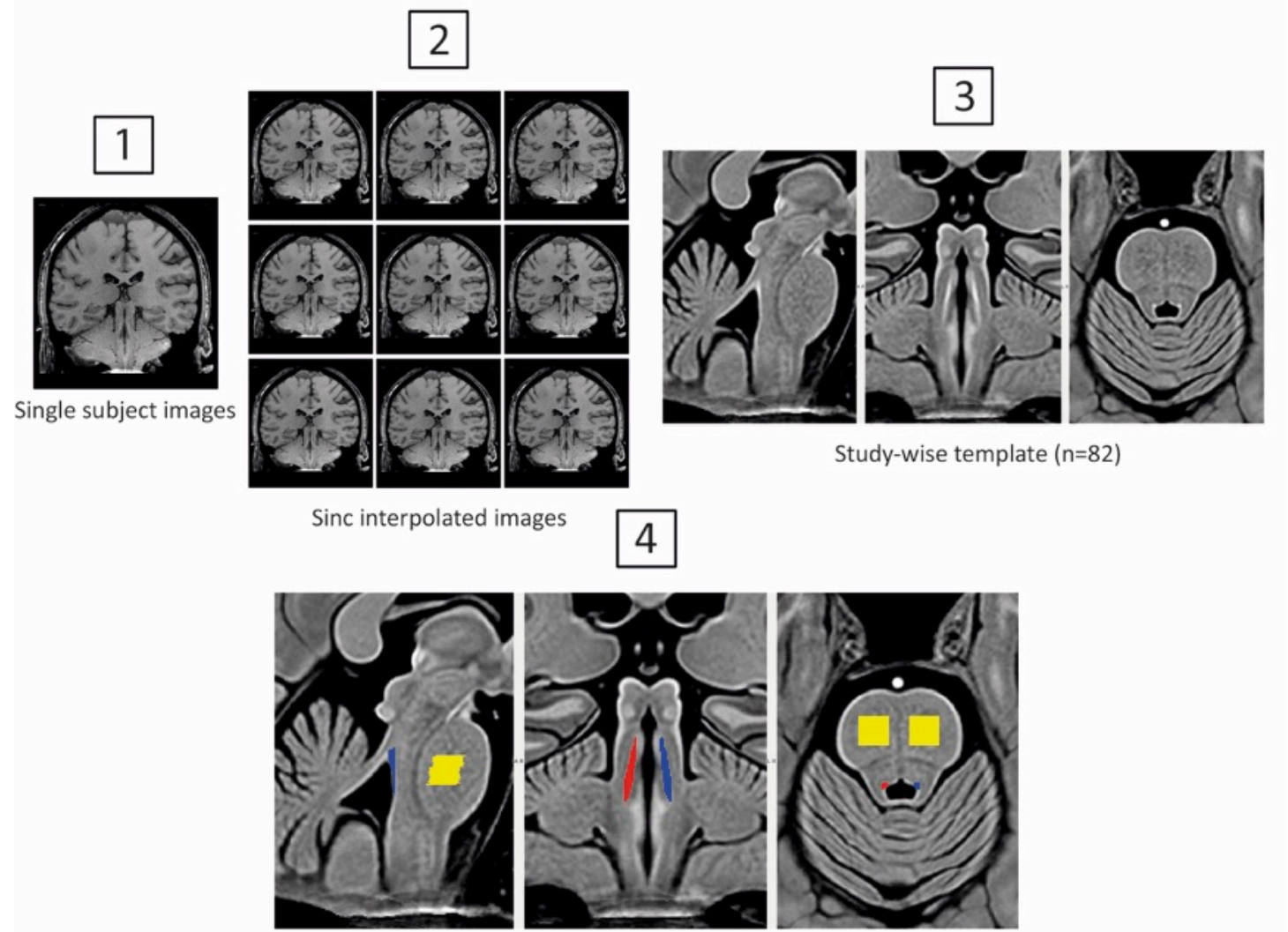

Segmented LC and REF regions in template space
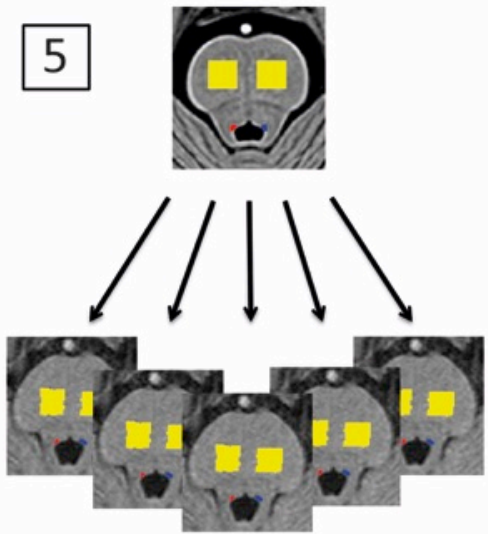

Template ROIs in single subject space

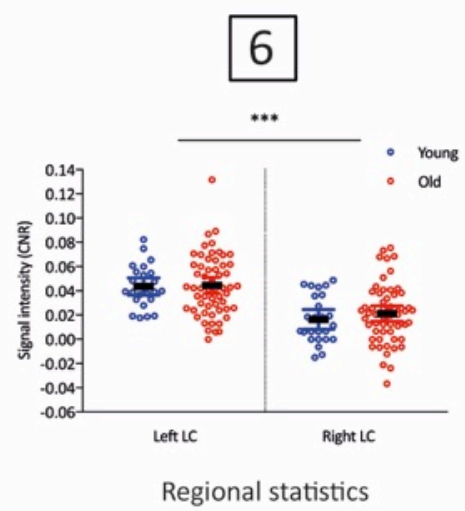

Figure 1. Summary steps of processing pipeline. $\mathrm{T}_{1}$-weighted FLASH images were sinc interpolated prior to normalisation. The locus coeruleus (LC) and reference regions of interest (ROIs) in the pons were manually segmented on the template image and then warped back to individual space. Extractions from single-subject LC and reference ROIs were subsequently used to generate contrast ratios and regional statistics. The numbering indicates the order of procedural steps. 
To assess the distribution of LC intensity voxels in young and older adults, the centre of mass was determined for each subject's LC mask at each axial slice and coordinates were warped to $0.5 \mathrm{~mm}^{3}$ MNI152 space (Montreal Neurological Institute, McGill University, Canada). MNI152 template space was chosen to facilitate comparisons with previously published LC signal intensity distributions i.e. as described by Keren et al., (Keren et al., 2009).

In order to assess LC signal intensity across the entire rostrocaudal axis, signal intensity profiles were estimated in single subject space to minimise partialvolume contamination. However, a common frame of reference was required in order to estimate group comparisons in LC signal intensity across subjects. To achieve this, the LC template mask delineated on the study-wise template was split at each axial slice spanning the entire mask from dorsal to ventral LC. Each axial slice in template space was subsequently warped to each single subject and intersected with each subjects LC mask. LC signal intensity values were determined from the resultant intersecting mask and compared across subjects. For all subjects, each axial slice was plotted in coordinates derived from the study-wise template.

\subsection{LC segmentation and signal intensity extractions}

The LC was manually segmented on each single-subject and study-wise template FLASH image using the software package ITK-SNAP (Yushkevich et al., 2006). On all images, the most superior LC slice in the axial plane was defined immediately after the inferior boundary of the interpeduncular fossa at the level of the inferior colliculus. The LC was segmented in the dorsal to ventral direction in the axial plane, typically as far as the most inferior slices in the $4^{\text {th }}$ ventricleanatomically at the level of the superior cerebellar peduncle. Each templatewarped LC mask was visually inspected and edited accordingly to ensure there were no spurious voxels in the $4^{\text {th }}$ ventricle. See Fig. 2 for example LC segmentations on a single subject $\mathrm{T}_{1}$-weighted FLASH image (A) and the studywise template (B).

A
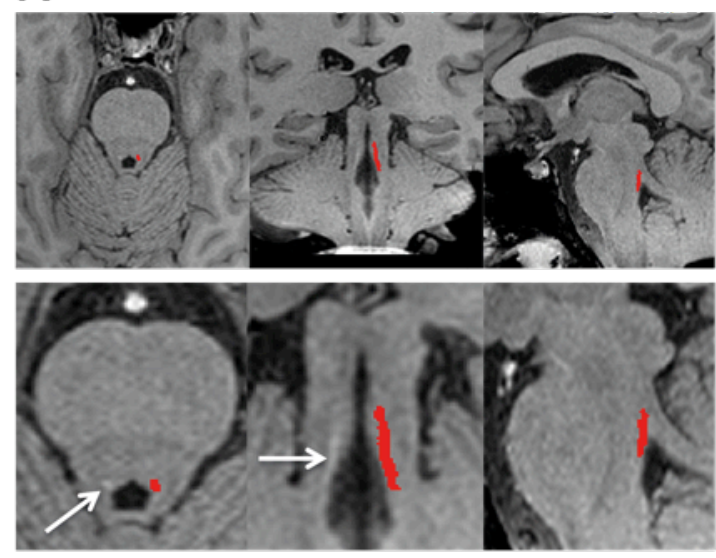

B

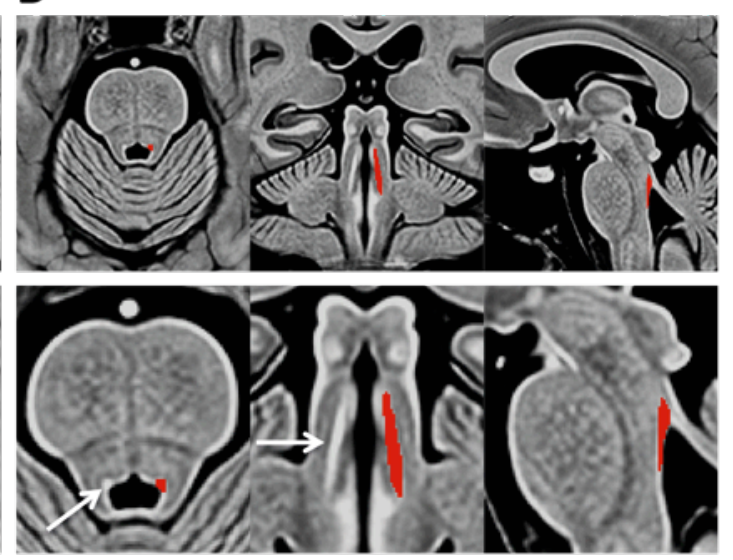


Figure 2. Example segmentations of the locus coeruleus (LC) region using $\mathrm{T}_{1^{-}}$ weighted FLASH MRI with interpolation. In (A) the LC manually segmented on a single-subject image and in (B) manually segmented on the study-wise template $(n=82)$.

To account for inter-slice and inter-subject variability, median and maximum signal intensities in left and right LC were assessed relative to reference regions delineated in the left and right rostral pontomesencephalic area as previously described (Garcia-Lorenzo et al., 2013). Reference regions ( $20 \times 20$ x 20 voxel) were segmented in the dorsal to ventral direction in the axial plane starting at a slice corresponding to the most dorsal aspect of the LC. Hence, all reported LC signal intensities are relative contrast ratios calculated using the following formula:

$$
\text { LC signal intensity }(\text { contrast })=\left(\mathrm{LC}_{\text {INT }}-\text { Pons }_{\text {INT }}\right) / \text { Pons }_{\text {INT }}
$$

\subsection{Validity and reliability of LC template based extractions}

In order to assess the validity of extracting quantitative information from an LC mask delineated directly in template space, median and maximum intensity values from each manually delineated single-subject LC mask were compared to values derived from an LC mask traced in template space. Single-measure, intraclass correlation coefficients (ICC) +/- the 95\% confidence interval (lower/upper bounds), taking the LC mask as class and correlating across extraction methods, demonstrated the proposed template based approach achieved very high accuracy when compared to manually delineated masks in single-subject space. For median and maximum LC extractions, ICC +/- the 95\% confidence interval was $0.89(0.84-0.93)$ and $0.93(0.89-0.95)$ respectively.

Test-retest reliability of median and maximum signal intensity values extracted from manually delineated LC masks was also evaluated using a single-measure ICC. For median and maximum LC extractions, ICC +/- the 95\% confidence interval was $0.92(0.87-0.95)$ and 0.96 (0.93 - 0.97) respectively, demonstrating that the proposed segmentation approach is highly reproducible.

Finally, to assess the reproducibility of signal intensity in the LC, 10 healthy young participants ( 5 male; 5 female) were scanned on a second occasion no longer than 3 months from the first measurement. Scan-rescan reproducibility of LC signal intensity was also assessed using a single-measure ICC. ICC +/- the $95 \%$ confidence for median and maximum signal intensity values was $0.63(0.04$ - 0.89) and $0.70(0.20-0.92)$, respectively, demonstrating neuromelaninsensitive FLASH imaging showed good reproducibility. 
Inter-hemispheric differences in LC signal intensity were probed using Wilcoxon rank-sum tests, which revealed a significant unilateral bias in left median ( $\mathrm{p}<$ $0.0001)$ and maximum $(\mathrm{p}<0.0001)$ signal intensity compared to right LC intensity values. A significant unilateral difference in median signal intensity was also observed in the left and right pons region $(\mathrm{p}<0.0001)$. Thus left and right median and maximum LC signal intensity values are reported separately throughout. No significant effect of sex was observed in left and right LC hemispheres for median and maximum signal intensity values (all $p>0.1$; Wilcoxon rank-sum tests). To probe age-related differences in LC signal intensity, old-versus-young Wilcoxon rank-sum statistics were computed using a statistical threshold of $\mathrm{p}<0.012$ correcting for multiple comparisons (i.e. Bonferroni-corrected $\mathrm{p}<0.05$ ). All tests were performed two-tailed.

\subsection{Computational framework}

Due to the size of the interpolated FLASH images and system memory required, the study-wise template was generated using a high-performance computer cluster comprising 44 nodes, each with 12 Cores, $2,53 \mathrm{GHz}$ and $190 \mathrm{~GB}$ of main memory requiring a processing time of 280 hours. All additional data postprocessing in MATLAB (R2014b; The Mathworks Inc., Natick, MA, USA) and FSL version 5.0 (http://fsl.fmrib.ox.ac.uk) were performed on a desktop computer with two, 3.2-GHz octa-core hyper-threading CPUs, 64 GB DDR3 RAM operated by Ubuntu Linux 14.04. Regional statistics were performed using the Statistical Package for the Social Sciences (SPSS), version 24.0.

\section{RESULTS}

\subsection{Anatomical location of the human LC in vivo}

An increase in $\mathrm{T}_{1}$-weighted FLASH signal intensity was observed caudally from the inferior colliculus extending to its most caudal aspect in the pons at the level of the superior cerebellar peduncle. To assess the topographical distribution of LC signal intensity, the centre of mass of all voxels within the LC mask at each rostrocaudal slice for each subject was determined and warped to MNI152 space. LC signal intensity distributions plotted from all adults $(n=82)$ revealed the LC was visible across a region spanning $22 \mathrm{~mm}$ (dorsal to ventral, $\mathrm{Z}=-15.5$ to -37.5 ; Fig. 3A). Assessment of LC signal intensity voxels in young $(n=25)$ and older adults ( $n=57)$ separately, revealed variance in the most dorsal slices corresponding to the most rostral LC were highly comparable between groups. In contrast older adults were more likely to demonstrate LC signal in the most ventral slice corresponding to the most caudal aspects of the LC. Note however fewer subjects exhibited a reliably observable signal in this most caudal LC region (Fig.3B). 
A

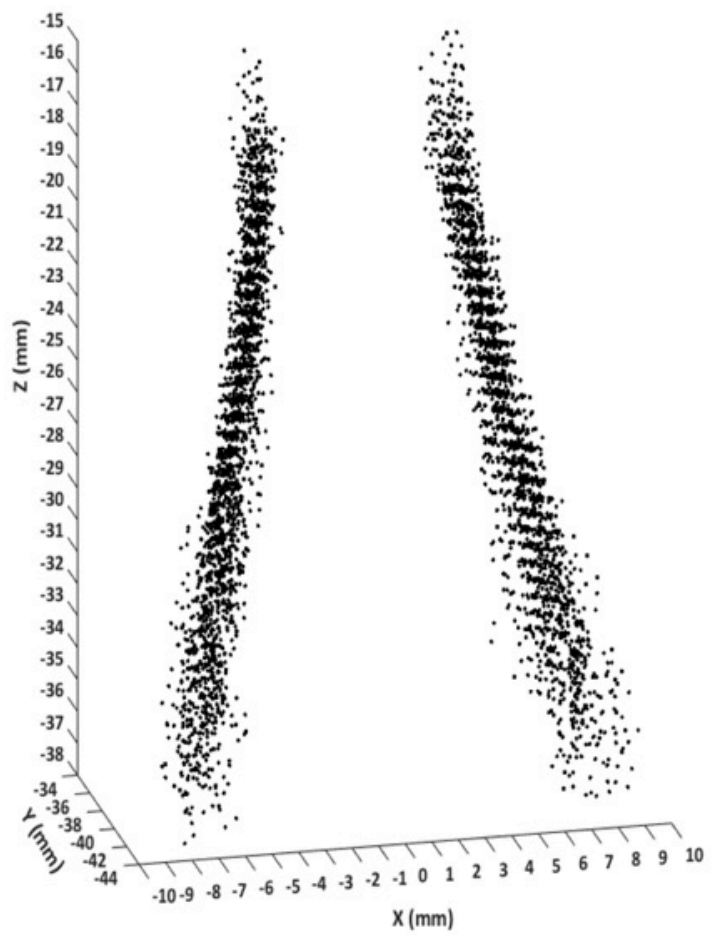

B

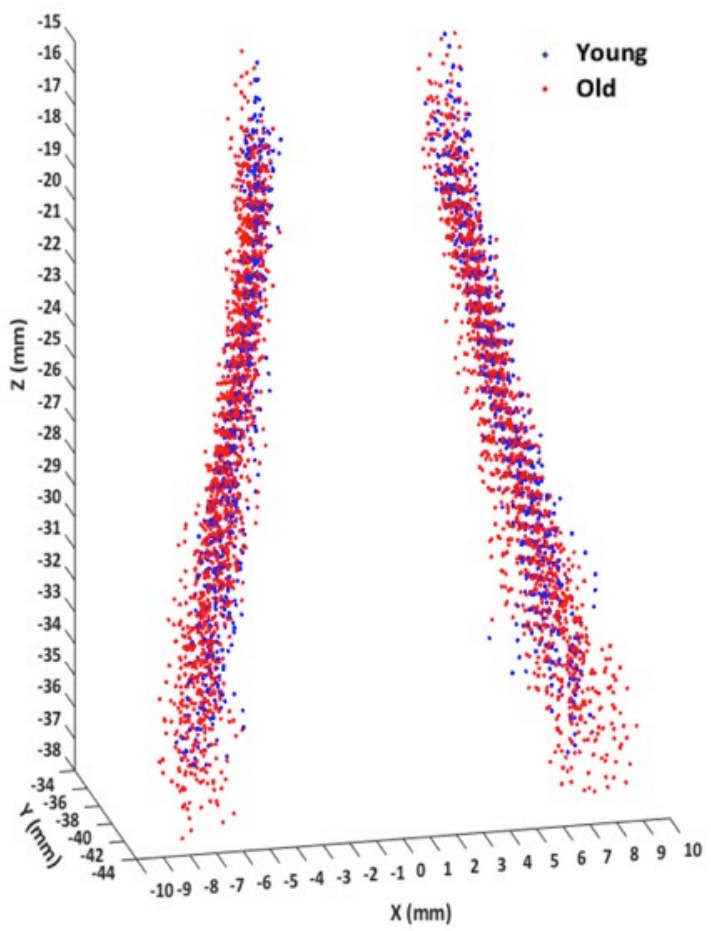

Figure 3. Topographical distribution of the human locus coeruleus (LC). Each point represents the centre of mass of all voxels within the LC mask at each rostrocaudal slice for (A) all participants $(n=82)$ and (B) young (blue) and older adults (red) plotted separately. Results presented were standardised to MNI152 space.

\subsection{Age-related differences in LC signal intensity}

In both young and older adults, significantly greater signal intensity was observed in left compared to right LC hemispheres for both median and maximum signal intensity values (Wilcoxon signed-ranks test; $\mathrm{p}<0.0001$ ). No significant age-related difference in LC median signal intensity was observed between young and older adults (Fig. 4A). However a significant age-related increase in maximum signal intensity was observed in left $(\mathrm{p}<0.01)$ and right $(\mathrm{p}<0.001)$ LC hemispheres (two-tailed Wilcoxon rank-sum test; Fig. 4B). No significant age-related difference was observed in left $(p>0.68)$ and right ( $p>0.84$ ) pons reference hemispheres (two-tailed Wilcoxon rank-sum test). 

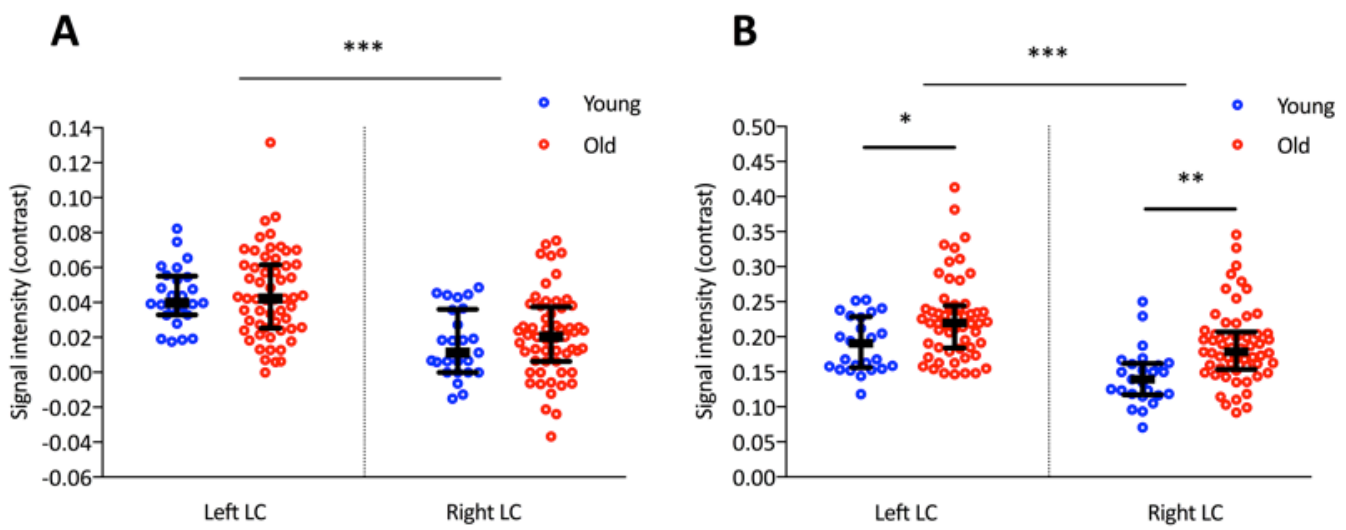

Figure 4. Summary of regional group statistics. (A) Median and (B) maximum + interquartile range signal intensity contrast ratios in left and right locus coeruleus (LC) of young and older adults. ${ }^{* * *}(\mathrm{p}<0.0001)$, indicates significant differences between left and right LC hemispheres (Wilcoxon signed-ranks test). ${ }^{* *}(\mathrm{p}<0.001),{ }^{*}(\mathrm{p}<0.01)$, indicate significant differences in old compared to younger adults (Wilcoxon ranked-sum tests).

\subsection{Topographical distribution of LC signal intensity}

Assessment of median and maximum signal intensity values from ventral to dorsal LC plotted in coordinates derived from study-wise template space, revealed a comparable signal intensity profile in left and right LC hemispheres. For median values, higher LC signal intensity in both left and right LC was observed in the most ventral third of the LC (peak signal intensity in both LC hemispheres at $\mathrm{Z}=88$; Fig. $5 \mathrm{~A}$ ). For maximum values, higher signal intensity in both left and right LC was observed in ventral but also middle to dorsal third of the LC (peak signal intensity at $\mathrm{Z}=115$ and $\mathrm{Z}=119$ respectively; Fig. 5B). Lowest median and maximum signal intensity values were observed in the most dorsal LC ( $\mathrm{Z}=129$ to 132$)$. Consistent with overall median and maximum intensity values, significantly greater signal intensity in left compared to right LC was observed across the entire rostrocaudal axis of the LC (both $\mathrm{p}<0.0001$; twotailed Wilcoxon signed-ranks test). 

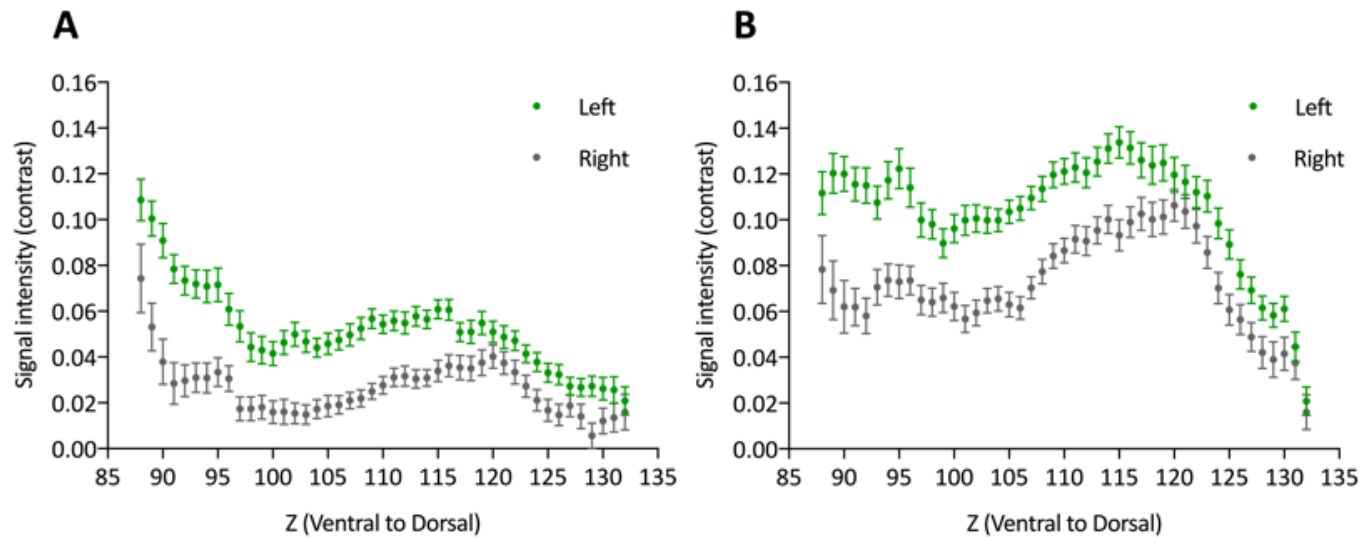

Figure 5. (A) Median and (B) maximum \pm SEM signal intensity contrast ratios in left (green) and right (grey) locus coeruleus (LC) hemispheres plotted at each axial (ventral to dorsal) slice in study-wise template space. Significantly greater median and maximum signal intensity was observed at each ventral to dorsal slice in left compared to right LC hemispheres (Wilcoxon signed-ranks test; $\mathrm{p}<$ 0.0001).

\subsection{Age-related differences in LC signal intensity distributions}

A largely concordant median signal intensity profile was observed in young and older adults in both left and right LC hemispheres (Figs. $6 \mathrm{~A}, \mathrm{~B}$ respectively). However an age-related increase in the dorsal third of the right LC was observed $(\mathrm{Z}=123$ to 128). Post-hoc Kruskal-Wallis tests revealed a significant age-related increase in right $\mathrm{LC}$ at $\mathrm{Z}=123$ and 128 (both $\mathrm{p}<0.007$; Bonferroni-corrected $\mathrm{p}<0.05)$. Age-related increases in maximum signal intensity were also observed at the dorsal third ( $\mathrm{Z}=123$ to 128) of both left and right LC hemispheres (Figs. 6 $\mathrm{C}, \mathrm{D}$ respectively). A significant age-related difference in signal intensity across the entire rostrocaudal axis of the LC was observed in the right LC hemisphere (two-tailed Wilcoxon rank-sum test: $p<0.01$ ). Post-hoc Kruskal-Wallis tests revealed a significant age-related increase in right maximum signal intensity at $\mathrm{Z}$ $=122$ to $124(\mathrm{p}<0.001)$ and $\mathrm{Z}=121,125,127,128$ (all $\mathrm{p}<0.007$; Bonferronicorrected $\mathrm{p}<0.05)$. A significant age-related increase in left maximum signal intensity was also observed at $\mathrm{Z}=121$ ( $\mathrm{p}<0.007$ ). Maximum signal intensity values were largely comparable in the ventral and middle third of both left and right LC hemispheres. 

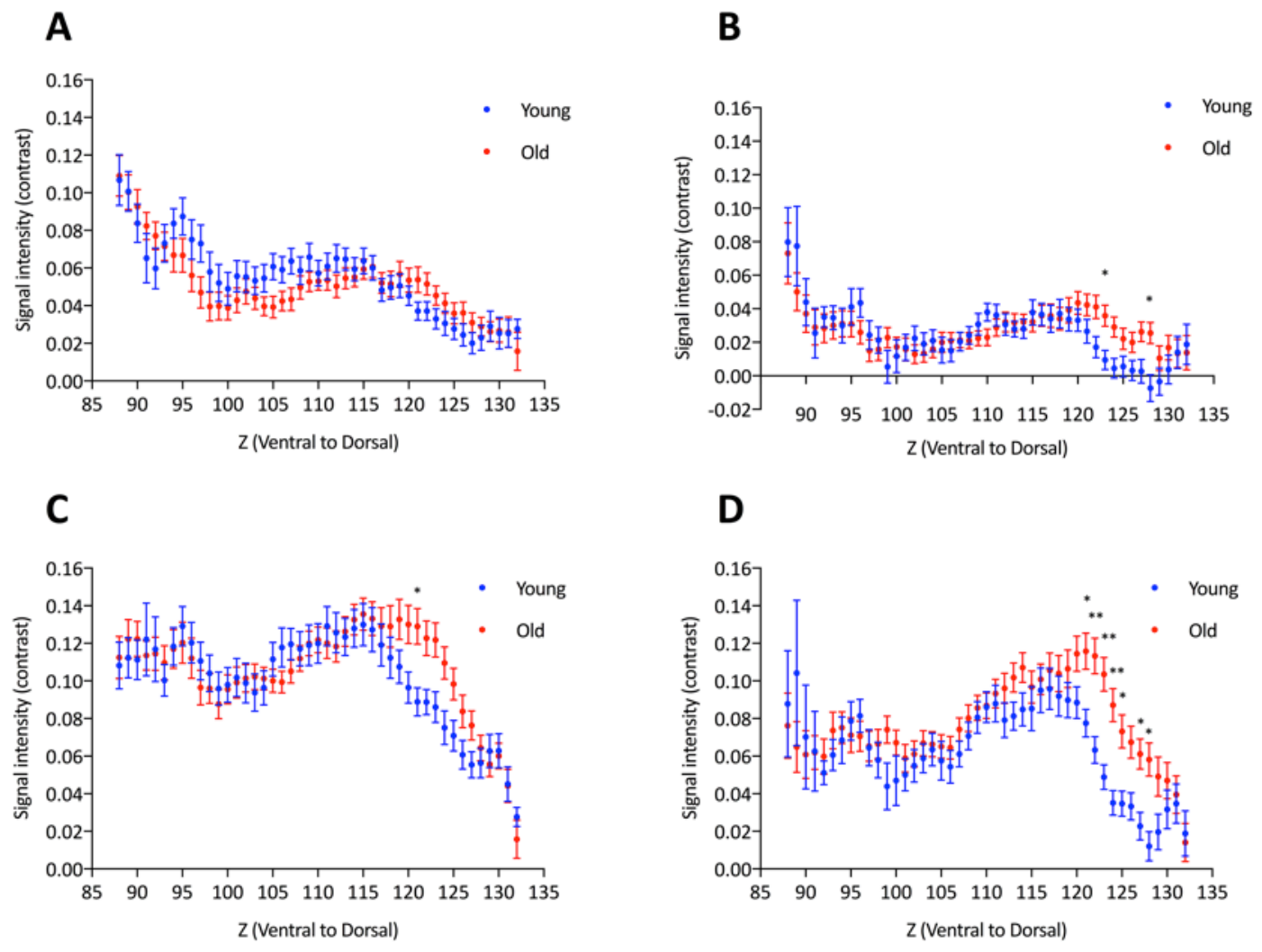

Figure 6. Median \pm SEM signal intensity contrast ratios in (A) left and (B) right locus coeruleus (LC) hemispheres of young (blue) and older (red) adults, from each axial (ventral to dorsal) slice plotted in coordinates from study-wise template space. Maximum \pm SEM signal intensity contrast ratios in (C) left and (D) right LC hemispheres of young (blue) and older (red) adults, from each axial (ventral to dorsal) slice plotted in coordinates from study-wise template space. A significant age-related difference in signal intensity across the entire rostrocaudal axis of the LC was observed in the right LC hemisphere (two-tailed Wilcoxon rank-sum test: $p<0.01) .{ }^{* *}(\mathrm{p}<0.001),{ }^{*}(\mathrm{p}<0.007$; Bonferroni-corrected $\mathrm{p}<0.05$ ), indicate significant differences in old compared to younger adults at each rostrocaudal slice (Post-hoc Kruskal-Wallis tests).

\section{DISCUSSION}

The purpose of this study was to investigate the topographical distribution of the human LC in young and older adults by measuring signal intensity changes, attributed to neuromelanin, using $\mathrm{T}_{1}$-weighted FLASH imaging at $3 \mathrm{~T}$. The highresolution isotropic acquisition achieved using FLASH imaging enabled us to characterise signal intensity changes across the entire rostrocaudal extent of the LC, providing first in vivo evidence that age-related changes in the LC are nonuniform. Furthermore, this study presents a standardised map of the human LC in vivo from 82 adults, which may facilitate further investigation into the structure and function of the LC in aging and neurodegenerative disease. 
Using $\mathrm{T}_{1}$-weighted FLASH imaging, an increase in signal intensity corresponding to the most dorsal aspect of the LC was observed immediately following the inferior boundary of the interpeduncular fossa at the level of the inferior colliculus and passed continuously ventral to an inferior point in the $4^{\text {th }}$ ventricle at the level of the trigeminal nerve. In all adults, independent of age, we observed higher signal intensity values in the middle to ventral third of both left and right LC hemispheres. Lowest median and maximum signal intensity values were noted in the dorsal third of the LC. Furthermore, the distribution of signal intensity voxels from all 82 participants in the study revealed an anatomically symmetrical LC structure spanning a region of $22 \mathrm{~mm}$ (MNI152 dorsal to ventral, $\mathrm{Z}=-15.5$ to -37.5$)$.

The spatial map of the LC reported here is highly concordant with that previously shown using TSE imaging (Keren et al., 2009). However in this study the LC region further extended in both the dorsal and ventral direction, which may be attributed to the higher resolution in the slice direction using FLASH imaging. Furthermore these findings closely resembled the 3D reconstructions of the LC obtained using serial histological sections of neuromelanin pigmented cells (German et al., 1988). Additionally it has been shown that the region of high signal intensity identified using in vivo MRI tightly correlated with the density of cells in the LC complex reported post-mortem (German et al., 1988), and that tyrosine hydroxylase (TH) neurons in the LC (which contain high levels of neuromelanin compared to other monoaminergic neurons) produced strong MRI contrast whereas similar TH neurons in dorsal raphe (lacking neuromelanin in histological stains) did not (Keren et al., 2015, 2009). These validation studies used $\mathrm{T}_{1}$ weighted TSE/FSE sequences with strong inherent magnetization transfer effects compared to $\mathrm{T}_{1}$ weighted FLASH imaging applied here. Nonetheless the good delineation of the LC reported in this study using $\mathrm{T}_{1}$ weighted FLASH suggests that $\mathrm{T}_{1}$ shortening is an important factor in driving LC contrast. Indeed, a recent study demonstrated that neuromelanin MRI contrast is driven primarily by $\mathrm{T}_{1}$-reduction owing to melanin-iron complexes and that magnetization transfer contrast is not significantly influenced by the presence of melanin (Trujillo et al., 2016). Collectively these findings provide convincing evidence that the region of increased signal intensity observed in vivo is an accurate marker of the number of neuromelanin pigmented cells in the LC.

The standardised map of the LC generated from this study may be used to probe the structure and function of the LC in vivo using additional modalities. To this end, two versions of an LC mask are provided in the online supplementary materials available for download. This includes an LC mask in study-wise template space, also provided, together with an LC mask warped to $0.5 \mathrm{~mm}^{3}$ MNI152 space. To reduce the effect of warping inaccuracies and minimise partial-volume contamination in the LC region, it is highly recommended to use the LC mask delineated in study-wise template space. However this will likely be dependent on the resolution and type of modality employed by the user. This may include using high-resolution fMRI to investigate the role of the LC in cognitive processes such as episodic memory (Düzel et al., 2015; Sterpenich et al., 2006), but may also facilitate investigations to assess the structural integrity of the LC i.e. in aging and neurodegenerative disease, using quantitative MRI 
techniques such as quantitative susceptibility mapping (QSM) and quantitative multi-parametric mapping (MPM) (Acosta-Cabronero et al., 2017; Betts et al., 2016; Callaghan et al., 2014).

An additional motivation of the study was to investigate how LC signal intensity changes may vary between healthy young and older adults. Overall we found LC signal intensity was markedly preserved, evidenced by no significant age-related increase in left or right median signal intensity values. It has been suggested that neuromelanin may increase with age until mid adulthood and decrease in late life (Manaye et al., 1983; Mann et al., 1983) which may correspond to an initial rise but decrease in LC signal intensity as suggested by Shibata et al., (2006). Since LC signal intensity was only assessed in older and not midlife adults, it may be that the lack of age-related differences in median signal intensity reported here may coincide with a potential reduction in neuromelanin in late life. However, a significant age-related increase in maximum signal intensity was observed in both left and right LC hemispheres suggesting age-related differences may be confined to regions with a high density of LC pigmented cells. Assessment of signal intensity changes across the entire rostrocaudal axis of the LC revealed age-related increases in the rostral third of the LC region. Additionally, no such age-related differences were observed in the caudal and middle third of the LC region, which taken together, may suggest age-related differences in neuromelanin are non-uniform.

The age-related increase in maximum LC signal intensity is consistent with previously reported in vivo MRI studies (Clewett et al., 2015; Shibata et al., 2006) and post-mortem findings demonstrating an age-related increase in neuromelanin (Zucca et al., 2006). Interestingly, the regional age-related difference in the rostral third of the LC is concordant with post-mortem studies identifying significant age-related reductions in neuromelanin-pigmented cells in the rostral portion of the nucleus (German et al., 1988; Manaye et al., 1995). However, these histological findings should be interpreted with caution since more recent studies using larger sample sizes, unbiased estimation procedures and excluding cases with pathology elsewhere in the brain have found no such age-related differences (Kubis et al., 2000; Mouton et al., 1994; Ohm et al., 1997; Theofilas et al., 2016). The morphology of LC cells has also been shown to change during aging, whereby studies have reported shrinkage of the perikarya and a subsequent decrease in cell size with advancing age (Chan-Palay \& Asan, 1989; Cui et al., 1988). Thus, one explanation for the age-related increase in LC signal intensity may be shrinkage of pigmented cells thereby increasing cell density within certain regions, however this remains entirely speculative at this time. Currently, the relationship between age-related increases in signal intensity identified in vivo and the morphological status, degree of cell loss reported postmortem, remains unclear and an important area to address in future research.

A previous study by Braak et al., (2011) has shown LC tau pathology may increase with age. Furthermore, it has been suggested phosphorylated tau in the LC may be the epicentre of pathology leading to AD since tau aggregates appear in the LC before tau lesions in the entorhinal cortex or the appearance of amyloid beta (Aß) pathology in the cortex (Braak et al., 2011). However Aß may also 
cause degeneration of LC neurons (Phillips et al., 2016), evidenced by the presence of LC damage in transgenic mice with robust amyloid burden (Kalinin et al., 2012) and observations that LC neurons that project to areas of high amyloid burden have reduced size (Braak and Del Tredici, 2012). Additionally, LC neurons are sensitive to the presence of environmental toxins, and increased levels of heavy metals (such as mercury, silver and bismuth) have been detected in the human LC (Pamphlett and Kum Jew, 2015). Circulating toxicants may enter the LC neurons selectively aided by the extensive exposure these neurons have to CNS capillaries, as well as by stress-induced upregulation of the nucleus (Pamphlett, 2014). Under normal conditions, neuromelanin can be neuroprotective by chelation of heavy metals (Double, 2012), however, age- or disease-related cumulative stress or toxin accumulation could prevent or reverse that chelation leading to damage. One striking neuroanatomical difference between human and most non-primate animals is the lack of neuromelanin in the LC of animals at any age, despite their LC neurons also producing catecholamines (Double et al., 2008). This raises an intriguing possibility that human-specific neurodegenerative diseases may be related to the presence of neuromelanin in the human LC.

To further understand the significance of age-related changes in neuromelanin, it will be essential to assess how LC signal intensity changes relate to cognition. It has been hypothesized a reduction in LC integrity and the related decline in noradrenergic modulation may contribute to cognitive decline during healthy aging (as reviewed by Mather \& Harley, 2016; Sara, 2009). Perhaps the most compelling evidence to date comes from an autopsy study conducted in 165 individuals from the Rush Memory and Aging Project, which found reduced postmortem neuronal density in the LC was associated with cognitive decline (based on a composite score of tests of episodic, semantic and working memory, perceptual speed, and visuospatial ability) approx. 6 years before death (Wilson et al., 2013). In vivo evidence for a relationship between LC structural integrity and cognition remains scarce, however a recent study demonstrated both verbal intelligence and a composite reserve score were positively associated with LC signal intensity in older adults. LC signal intensity was also more strongly associated with attentional shifting ability in older adults with lower cognitive reserve suggesting in vivo estimates of LC neuromelanin signal intensity may be linked to cognitive reserve in normal aging (Clewett et al., 2015).

It is hoped the advancement of neuromelanin-sensitive MRI to investigate the LC in vivo may help to elucidate why the LC is especially vulnerable to damage in neurodegenerative disease. Recent evidence would suggest a topographical vulnerability across disorders, whereby the rostral and middle thirds of the LC may be prone to greater cell loss in AD (Theofilas et al., 2016), whilst extensive neuronal loss across the entire length of the LC is seen in PD (Vermeiren and De Deyn, 2017). The regional neuronal cell loss in AD has additional significance in that studies in rodents and monkeys have shown the rostral and middle thirds of the LC project to and receive inputs from cortical, diencephalic, and forebrain structures found especially vulnerable to AD in humans (Marcyniuk et al., 1986; (Samuels and Szabadi, 2008b) Loughlin et al., 1986). On the contrary, the caudal third of the LC is reciprocally connected with regions unaffected in AD such as 
the spinal cord and cerebellum (Westlund \& Coulter, 1980). Future work will seek to identify if these findings can be corroborated in vivo and will be critical in establishing neuromelanin-sensitive MRI as a surrogate marker of LC structure.

Furthermore, the findings presented here taken together with the surrounding literature, stress the importance of future studies in aging and disease to assess regional sub-divisions of the LC to more precisely understand the structure and function of the human LC.

It is important to note several limitations on interpretation of these findings. First, we can only infer age-related differences from a cross-sectional study and cannot comment on lifespan trajectories of neuromelanin deposition. It will be key for future longitudinal studies to assess if age-related accumulation of neuromelanin in the LC follows a similar topographical profile to the findings reported in this study. Second, a significant unilateral bias in both the LC and reference signal intensity values was observed as noted by other MRI studies in aging and also PD (Garcia-Lorenzo et al., 2013; Keren et al., 2009). However post-mortem studies, have reported a largely symmetrical distribution of neuromelanin pigmented cells (Baker et al., 1989; German et al., 1988; ChanPalay and Asan, 1989; Ohm et al.,1997), which taken together, suggests that the observed unilateral bias is caused by interaction of the transmit radio frequency field with the human head and the signal reception profile of the multi-channel head coils. In support of this notion, a recent study using a 3T Philips MRI scanner also found a unilateral asymmetry in the LC yet the opposite to that reported here i.e. higher signal intensity contrast in right compared to left hemispheres (Tona et al., 2017). The $\mathrm{B}_{0}$-field direction in 3T Philips-MRscanners is opposite to that of the Siemens-MR-scanners, thus the left/right switch in signal asymmetry between Siemens and Philips vendors is a strong indication that opposing directions in rotation of $\mathrm{B}_{1}$ fields relative to the head may underlie these discrepancies. Third, the reproducibility in signal intensity reported here was lower than that shown in a recent study conducted on the same day (Langley et al., 2016). It remains to be determined whether scanner performance and subject physiological status may have influenced signal intensity reproducibility using scans conducted on different days. Finally, a general limitation of the current and similarly reported LC-MRI studies (Clewett et al., 2015; Garcia-Lorenzo et al., 2013; Keren et al., 2009; Matsuura et al., 2013; Ohtsuka et al., 2013; Sasaki et al., 2009, 2006; Schwarz et al., 2011; Shibata et al., 2006 ) is that all have analysed signal intensity on $\mathrm{T}_{1}$-weighted images and not quantitative MRI data. As such, semi-quantitative methods (e.g., using contrast with respect to an adjacent reference region) are necessary to investigate group comparisons using $\mathrm{T}_{1}$-weighted data. The concern in taking this approach forward is that tissue serving as the reference region may be atypical in neurodegenerative disorders. Quantitative imaging methods are necessary to avoid these confounds, and are eagerly awaited to identify the full potential of neuromelanin-sensitive imaging as a suitable biomarker of LC degeneration. 


\section{CONCLUSIONS}

In vivo high-resolution $\mathrm{T}_{1}$-weighted FLASH imaging at $3 \mathrm{~T}$ is an effective tool to localise the human LC in young and older adults. This study provides first in vivo evidence characterising the topographical distribution of LC signal intensity changes across the entire rostrocaudal length of the LC revealing age-related differences are non-uniform. The standardised template mask of the LC generated from 82 adults in this study may be used to further probe the cognitive significance of signal intensity changes attributed to neuromelanin. The findings presented stress the importance of future studies to assess regional subdivisions of the LC to more precisely understand how the structure and function of the human LC may be differentially affected in aging and neurodegenerative disease.

\section{ACKWNOWLDEGMENTS}

We thank Urte Schneider, Christin Russ, Iris Mann, Anne Hochkeppler and our local radiographers Kerstin Möhring and Ilona Wiedenhöft, for help in subject recruitment and data collection. We also thank Julio Acosta-Cabronero, Lea Grinberg and her colleagues for helpful discussions during preparation of the manuscript. This work was supported by the SFB 779-TP A07 awarded to E.D. 


\section{REFERENCES}

Acosta-Cabronero, J., Betts, M.J., Cardenas-Blanco, A., Yang, S., Nestor, P.J., 2016. In Vivo MRI Mapping of Brain Iron Deposition across the Adult Lifespan. J. Neurosci. 36, 364-374. doi:10.1523/JNEUROSCI.1907-15.2016

Acosta-Cabronero, J., Cardenas-Blanco, A., Betts, M.J., Butryn, M., Valdes-Herrera, J.P., Galazky, I., Nestor, P.J., 2017. The whole-brain pattern of magnetic susceptibility perturbations in Parkinson's disease. Brain 140, 118-131. doi:10.1093/brain/aww278

Aston-Jones, G., Bloom, F.E., 1981. Activity of norepinephrine-containing locus coeruleus neurons in behaving rats anticipates fluctuations in the sleepwaking cycle. J Neurosci 1, 876-886.

Aston-Jones, G., \& Cohen, J.D., 2005. An integrative theory of locus coeruleusnorepinephrine function: adaptive gain and optimal performance. Annu. Rev. Neurosci. 28, 403-450.

Avants, B., Epstein, C., Grossman, M., Gee, J., 2008. Symmetric diffeomorphic image registration with cross-correlation: Evaluating automated labeling of elderly and neurodegenerative brain. Med. Image Anal. 12, 26-41. doi:10.1016/j.media.2007.06.004

Baker, K.G., Törk, I., Hornung, J.-P., Halasz, P., 1989. The human locus coeruleus complex: an immunohistochemical and three dimensional reconstruction study. Exp. Brain Res. 77, 257-270.

Benarroch, E.E., 2009. The locus ceruleus norepinephrine system Functional organization and potential clinical significance. Neurology 73, 1699-1704.

Betts, M.J., Acosta-Cabronero, J., Cardenas-Blanco, A., Nestor, P.J., Düzel, E., 2016. High-resolution characterisation of the aging brain using simultaneous quantitative susceptibility mapping (QSM) and R2* measurements at 7T. NeuroImage 138, 43-63. doi:10.1016/j.neuroimage.2016.05.024

Braak, H., Del Tredici, K., 2015. The preclinical phase of the pathological process underlying sporadic Alzheimer's disease. Brain 138, 2814-2833. doi:10.1093/brain/awv236

Braak, H., Del Tredici, K., 2012. Where, when, and in what form does sporadic Alzheimer's disease begin?: Curr. Opin. Neurol. 25, 708-714. doi:10.1097/WC0.0b013e32835a3432

Braak, H., Del Tredici, K., Rüb, U., de Vos, R.A., Steur, E.N.J., Braak, E., 2003. Staging of brain pathology related to sporadic Parkinson's disease. Neurobiol. Aging 24, 197-211.

Braak, H., Thal, D.R., Ghebremedhin, E., Del Tredici, K., 2011. Stages of the pathologic process in Alzheimer disease: age categories from 1 to 100 years. J. Neuropathol. Exp. Neurol. 70, 960-969.

Callaghan, M.F., Freund, P., Draganski, B., Anderson, E., Cappelletti, M., Chowdhury, R., Diedrichsen, J., FitzGerald, T.H.B., Smittenaar, P., Helms, G., Lutti, A., Weiskopf, N., 2014. Widespread age-related differences in the human brain microstructure revealed by quantitative magnetic resonance imaging. Neurobiol. Aging. doi:10.1016/j.neurobiolaging.2014.02.008

Chan-Palay, V., \& Asan, E., 1989. Quantitation of catecholamine neurons in the locus coeruleus in human brains of normal young and older adults and in depression. J. Comp. Neurol. 287, 357-372. 
Clewett, D.V., Lee, T.-H., Greening, S., Ponzio, A., Margalit, E., Mather, M., 2015. Neuromelanin marks the spot: identifying a locus coeruleus biomarker of cognitive reserve in healthy aging. Neurobiol. Aging. doi:10.1016/j.neurobiolaging.2015.09.019

Cui, Z. T., Zhang, T. M., Su, Z. H., Yen, W. W., 1988. Morphological changes in locus coeruleus of albino rats in relation to aging. Acta Anat. 131, 207-209.

Dickson, D.W., Fujishiro, H., DelleDonne, A., Menke, J., Ahmed, Z., Klos, K.J., Josephs, K.A., Frigerio, R., Burnett, M., Parisi, J.E., Ahlskog, J.E., 2008. Evidence that incidental Lewy body disease is pre-symptomatic Parkinson's disease. Acta Neuropathol. (Berl.) 115, 437-444. doi:10.1007/s00401-008-0345-7

Double, K. L., 2012. Neuronal vulnerability in Parkinson's disease. Parkinsonism Relat. Disord.18 (Suppl 1), S52-S54.

Double, K.L., Dedov, V.N., Fedorow, H., Kettle, E., Halliday, G.M., Garner, B., Brunk, U.T., 2008. The comparative biology of neuromelanin and lipofuscin in the human brain. Cell. Mol. Life Sci. 65, 1669-1682. doi:10.1007/s00018-0087581-9

Düzel, E., Guitart-Masip, M., Maass, A., Hämmerer, D., Betts, M.J., Speck, O., Weiskopf, N., Kanowski, M., 2015. Midbrain fMRI: Applications, Limitations and Challenges, in: Uludag, K., Ugurbil, K., Berliner, L. (Eds.), FMRI: From Nuclear Spins to Brain Functions. Springer US, Boston, MA, pp. 581-609.

Enochs, W.S., Hyslop, W.B., Bennett, H.F., Brown III, R.D., Koenig, S.H., Swartz, H.M.,1989. Sources of the increased longitudinal relaxation rates observed in melanotic melanoma. An in vitro study of synthetic melanins. Invest. Radiol. 24, 794-804.

Enochs, W.S., Petherick, P., Bogdanova, A., Mohr, U., Weissleder, R., 1997. Paramagnetic metal scavenging by melanin: MR imaging. Radiology 204, 417-423.

Espay, A.J., LeWitt, P.A., Kaufmann, H., 2014. Norepinephrine deficiency in Parkinson's disease: The case for noradrenergic enhancement: Norepinephrine Deficiency in PD. Mov. Disord. 29, 1710-1719. doi:10.1002/mds.26048

Garcia-Lorenzo, D., Longo-Dos Santos, C., Ewenczyk, C., Leu-Semenescu, S., Gallea, C., Quattrocchi, G., Pita Lobo, P., Poupon, C., Benali, H., Arnulf, I., Vidailhet, M., Lehericy, S., 2013. The coeruleus/subcoeruleus complex in rapid eye movement sleep behaviour disorders in Parkinson's disease. Brain 136, 2120-2129. doi:10.1093/brain/awt152

German, D.C., Manaye, K.F., White, C.L., Woodward, D.J., McIntire, D.D., Smith, W.K., Kalaria, R.N., Mann, D.M., 1992. Disease-specific patterns of locus coeruleus cell loss. Ann. Neurol. 32, 667-676.

German, D.C., Walker, B.S., Manaye, K., Smith, W.K., Woodward, D.J., North, A.J., 1988. The human locus coeruleus: computer reconstruction of cellular distribution. J. Neurosci. 8, 1776-1788.

Kalinin, S., Polak, P.E., Lin, S.X., Sakharkar, A.J., Pandey, S.C., Feinstein, D.L., 2012. The noradrenaline precursor L-DOPS reduces pathology in a mouse model of Alzheimer's disease. Neurobiol. Aging 33, 1651-1663. doi:10.1016/j.neurobiolaging.2011.04.012 
Kubis, N., Faucheux, B. A., Ransmayr, G., Damier, P., Duyckaerts, C., Henin, D., Forette, B., Le Charpentier, Y., Hauw, J.J., Agid, Y., Hirsch, E.C., 2000. Preservation of midbrain catecholaminergic neurons in very old human subjects. Brain. 123, 366-373.

Keren, N.I., Lozar, C.T., Harris, K.C., Morgan, P.S., Eckert, M.A., 2009. In vivo mapping of the human locus coeruleus. NeuroImage 47, 1261-1267. doi:10.1016/j.neuroimage.2009.06.012

Keren, N.I., Taheri, S., Vazey, E.M., Morgan, P.S., Granholm, A.-C.E., Aston-Jones, G.S., Eckert, M.A., 2015. Histologic validation of locus coeruleus MRI contrast in post-mortem tissue. NeuroImage 113, 235-245. doi:10.1016/j.neuroimage.2015.03.020

Langley, J., Huddleston, D.E., Liu, C.J., Hu, X., 2016. Reproducibility of locus coeruleus and substantia nigra imaging with neuromelanin sensitive MRI. Magn. Reson. Mater. Phys. Biol. Med. doi:10.1007/s10334-016-0590-z

Lohr, J.B., Jeste, D.V., 1988. Locus ceruleus morphometry in aging and schizophrenia. Acta Psychiatr. Scand. 77, 689-697.

Loughlin, S.E., Foote, S.L., Bloom, F.E., 1986. Efferent projections of nucleus locus coeruleus: topographic organization of cells of origin demonstrated by three-dimensional reconstruction. Neuroscience. 18:291-306.

Manaye, K.F., McIntire, D.D., Mann, D., German, D.C., 1995. Locus coeruleus cell loss in the aging human brain: A non-random process. J. Comp. Neurol. 358, 79-87.

Marcyniuk, B., Mann, D.M., Yates, P.0., 1986. Loss of nerve cells from locus coeruleus in Alzheimer's disease is topographically arranged. Neurosci Lett. 64, 247-252.

Mather, M., Harley, C.W., 2016. The Locus Coeruleus: Essential for Maintaining Cognitive Function and the Aging Brain. Trends Cogn. Sci. 20, 214-226. doi:10.1016/j.tics.2016.01.001

Matsuura, K., Maeda, M., Yata, K., Ichiba, Y., Yamaguchi, T., Kanamaru, K., Tomimoto, H., 2013. Neuromelanin Magnetic Resonance Imaging in Parkinson's Disease and Multiple System Atrophy. Eur. Neurol. 70, 70-77. doi:10.1159/000350291

Mouton, P.R., Pakkenberg, B., Jorgen, H., Gundersen, G., Price, D.L., 1994. Absolute number and size of pigmented locus coeruleus neurons in young and aged individuals. J. Chem. Neuroanat. 7, 185-190.

Ohm, T.G., Busch, C., Bohl, J., 1997. Unbiased estimation of neuronal numbers in the human nucleus coeruleus during aging. Neurobiol. Aging 18, 393-399.

Ohtsuka, C., Sasaki, M., Konno, K., Koide, M., Kato, K., Takahashi, J., Takahashi, S., Kudo, K., Yamashita, F., Terayama, Y., 2013. Changes in substantia nigra and locus coeruleus in patients with early-stage Parkinson's disease using neuromelanin-sensitive MR imaging. Neurosci. Lett. 541, 93-98. doi:10.1016/j.neulet.2013.02.012

Pamphlett, R., 2014. Uptake of environmental toxicants by the locus ceruleus: A potential trigger for neurodegenerative, demyelinating and psychiatric disorders. Med. Hypotheses 82, 97-104. doi:10.1016/j.mehy.2013.11.016

Pamphlett, R., Kum Jew, S., 2015. Different populations of human locus ceruleus neurons contain heavy metals or hyperphosphorylated Tau: implications for amyloid- $\beta$ and tau pathology in Alzheimer's disease. J. Alzheimers Dis. $45,437-447$. 
Phillips, C., Fahimi, A., Das, D., Mojabi, F. S., Ponnusamy, R., Salehi, A., 2016. Noradrenergic system in Down syndrome and Alzheimer's disease a target for therapy. Curr. Alzheimer Res. 13, 68-83.

Robbins, T. W., 1984. Cortical noradrenaline, attention and arousal. Psychol. Med. $14,13-21$.

Samuels, E.R., Szabadi, E., 2008a. Functional neuroanatomy of the noradrenergic locus coeruleus: its roles in the regulation of arousal and autonomic function part II: physiological and pharmacological manipulations and pathological alterations of locus coeruleus activity in humans. Curr. Neuropharmacol. 6, 254-285.

Samuels, E.R., Szabadi, E., 2008b. Functional neuroanatomy of the noradrenergic locus coeruleus: its roles in the regulation of arousal and autonomic function part I: principles of functional organisation. Curr. Neuropharmacol. 6, 235-253.

Sara, S.J., 2009. The locus coeruleus and noradrenergic modulation of cognition. Nat. Rev. Neurosci. 10, 211-223. doi:10.1038/nrn2573

Sasaki, M., Shibata, E., Ohtsuka, K., Endoh, J., Kudo, K., Narumi, S., Sakai, A., 2009. Visual discrimination among patients with depression and schizophrenia and healthy individuals using semiquantitative color-coded fast spin-echo T1-weighted magnetic resonance imaging. Neuroradiology 52, 83-89. doi:10.1007/s00234-009-0595-7

Sasaki, M., Shibata, E., Tohyama, K., Takahashi, J., Otsuka, K., Tsuchiya, K., Takahashi, S., Ehara, S., Terayama, Y., Sakai, A., 2006. Neuromelanin magnetic resonance imaging of locus ceruleus and substantia nigra in Parkinson's disease. Neuroreport 17, 1215-1218.

Schwarz, S.T., Rittman, T., Gontu, V., Morgan, P.S., Bajaj, N., Auer, D.P., 2011. T1Weighted MRI shows stage-dependent substantia nigra signal loss in Parkinson's disease. Mov. Disord. 26, 1633-1638. doi:10.1002/mds.23722

Shibata, E., Sasaki, M., Tohyama, K., Kanbara, Y., Otsuka, K., Ehara, S., Sakai, A., 2006. Age-related Changes in Locus Ceruleus on Neuromelanin Magnetic Resonance Imaging at 3 Tesla. Magn. Reson. Med. Sci. 5, 197-200. doi:10.2463/mrms.5.197

Sterpenich, V., D'Argembeau, A., Desseilles, M., Balteau, E., Albouy, G., Vandewalle, G., Degueldre, C., Luxen, A., Collette, F., Maquet, P., 2006. The Locus Ceruleus Is Involved in the Successful Retrieval of Emotional Memories in Humans. J. Neurosci. 26, 7416-7423. doi:10.1523/JNEUROSCI.1001-06.2006

Takahashi, J., Shibata, T., Sasaki, M., Kudo, M., Yanezawa, H., Obara, S., Kudo, K., Ito, K., Yamashita, F., Terayama, Y., 2015. Detection of changes in the locus coeruleus in patients with mild cognitive impairment and Alzheimer's disease: High-resolution fast spin-echo T1-weighted imaging: Locus coeruleus in cognitive impairment. Geriatr. Gerontol. Int. 15, 334-340. doi:10.1111/ggi.12280

Theofilas, P., Ehrenberg, A.J., Dunlop, S., Alho, A.T., Nguy, A., Paraizo Leite, R.E., Rodriguez, R.D., Mejia, M.B., Suemoto, C.K., de Lucena Ferretti-Rebustini, R.E., Polichiso, L., Nascimento, C.F., Seeley, W.W., Nitrini, R., Pasqualucci, C.A., Jacob Filho, W., Rueb, U., Neuhaus, J., Heinsen, H., Grinberg, L.T., 2016. Locus coeruleus volume and cell population changes during Alzheimer's 
disease progression: A stereological study in human postmortem brains with potential implication for early-stage biomarker discovery. Alzheimers Dement. doi:10.1016/j.jalz.2016.06.2362

Tomlinson, B., Irving, D., Blessed, G., 1981. Cell loss in the locus coeruleus in senile dementia of Alzheimer type. J. Neurol. Sci. 49, 419-428.

Tona, K.-D., Keuken, M.C., de Rover, M., Lakke, E., Forstmann, B.U., Nieuwenhuis, S., van Osch, M.J.P., 2017. In vivo visualization of the locus coeruleus in humans: quantifying the test-retest reliability. Brain Struct. Funct. doi:10.1007/s00429-017-1464-5

Trujillo, P., Summers, P.E., Ferrari, E., Zucca, F.A., Sturini, M., Mainardi, L.T., Cerutti, S., Smith, A.K., Smith, S.A., Zecca, L., Costa, A., 2016. Contrast mechanisms associated with neuromelanin-MRI: Neuromelanin-MRI Contrast. Magn. Reson. Med. doi:10.1002/mrm.26584

Vermeiren, Y., De Deyn, P.P., 2017. Targeting the norepinephrinergic system in Parkinson's disease and related disorders: The locus coeruleus story. Neurochem. Int. 102, 22-32. doi:10.1016/j.neuint.2016.11.009

Vijayashankar, N., \& Brody, H., Quantitative study of the pigmented neurons in the nuclei locus coeruleus and subcoeruleus in man as related to aging. J. Neuropathol. Exp. Neurol. 38, 490-497.

Wakamatsu, K., Tabuchi, K., Ojika, M., Zucca, F.A., Zecca, L., Ito, S., 2015. Norepinephrine and its metabolites are involved in the synthesis of neuromelanin derived from the locus coeruleus. J. Neurochem. 135, 768776. doi:10.1111/jnc.13237

Westlund, K.N., Coulter, J.D., 1980. Descending projections of the locus coeruleus and subcoeruleus/medial parabrachial nuclei in monkey: axonal transport studies and dopamine-beta-hydroxylase immunocytochemistry. Brain Res. 2, 235-64.

Wilson, R.S., Nag, S., Boyle, P.A., Hizel, L.P., Yu, L., Buchman, A.S., Schneider, J.A., Bennett, D.A., 2013. Neural reserve, neuronal density in the locus ceruleus, and cognitive decline. Neurology 80, 1202-1208.

Yushkevich, P.A., Piven, J., Hazlett, H.C., Smith, R.G., Ho, S., Gee, J.C., Gerig, G., 2006. User-guided 3D active contour segmentation of anatomical structures: Significantly improved efficiency and reliability. Neurolmage 31, 11161128. doi:10.1016/j.neuroimage.2006.01.015

Zucca, F.A., Bellei, C., Giannelli, S., Terreni, M.R., Gallorini, M., Rizzio, E., Pezzoli, G., Albertini, A., Zecca, L., 2006. Neuromelanin and iron in human locus coeruleus and substantia nigra during aging: consequences for neuronal vulnerability. J. Neural Transm. 113, 757-767. doi:10.1007/s00702-0060453-2

Zucca, F.A., Segura-Aguilar, J., Ferrari, E., Muñoz, P., Paris, I., Sulzer, D., Sarna, T., Casella, L., Zecca, L., 2015. Interactions of iron, dopamine and neuromelanin pathways in brain aging and Parkinson's disease. Prog. Neurobiol. doi:10.1016/j.pneurobio.2015.09.012 